\title{
An Embedded Multiple Case Study: Facilitating Uptake of Food Insecurity Screening Initiatives in Low Resource Primary Care Settings
}

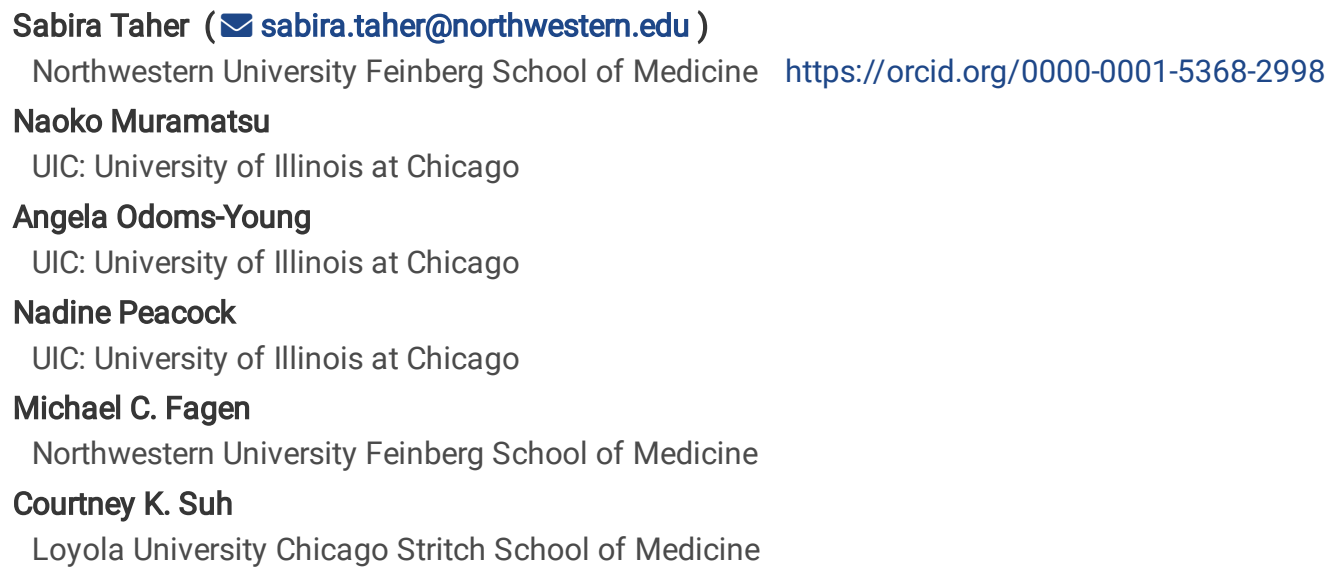

\section{Research Article}

Keywords: Food insecurity, food security screening, Implementation, Dissemination, Consolidated Framework for Implementation Research, Primary care practice, Semi-structured interviews, Produce prescription programs

Posted Date: May 10th, 2021

DOI: https://doi.org/10.21203/rs.3.rs-499136/v1

License: (c) (i) This work is licensed under a Creative Commons Attribution 4.0 International License. Read Full License 


\section{Abstract \\ Background}

Food insecurity (FI), limited access to healthy food, is a social determinant of health linked to poor dietary health and difficulty with disease management. Healthcare experts support the adoption of validated screening tools within primary care clinics to identify and connect FI patients to food resources. Yet, a lack of standard practices limits uptake. The purpose of this study was to understand program processes and outcomes of primary care focused FI screening initiatives that can guide wide-scale program implementation.

\section{Methods}

This was an embedded multiple case study of two primary care-focused initiatives implemented in two diverse health systems in Chicago and Suburban Cook County that routinely screened patients for $\mathrm{Fl}$ and referred them to onsite food assistance programs. The Consolidated Framework for Implementation Research and an iterative process were used to collect/analyze qualitative data through semi-structured interviews with $\mathrm{N}=19$ clinical staff. Intended program activities, outcomes, actors, implementation barriers/facilitators and overarching implementation themes were identified as a part of a cross-case analysis.

\section{Findings:}

Programs outcomes included: the number of patients screened and identified as Fl; the number of patients referred to and participated in the onsite food assistance program. Overall, neither case achieved intended reach or participation. Limited financial, human and infrastructure support were implementation barriers. Existing community collaborations and clinic-level autonomy were critical facilitators during implementation that contributed to the flexibility of program activities. Programs were tailored to each context to assist in implementation feasibility, but contributed to low program fidelity.

\section{Conclusions}

Programs were highly adaptable and each context enhanced implementation feasibility across settings. These characteristics have the potential to support program uptake in other settings, but should be used with caution to preserve program fidelity and to achieve intended outcomes. A foundational model for the development and testing of standard clinical practice was the product of this study.

\section{Contributions To The Literature}

- Research supports the implementation of validated food insecurity screening tools, combined with referrals and linkages to emergency food resources, in primary care settings to address food insecurity and assist with disease management. However, limited theoretically derived practices exist in the literature that can guide program implementation.

- We applied a broad implementation science framework, CFIR, to develop a formative conceptual model that can guide the development of standard practices.

- The findings contribute to gaps in theory-derived food insecurity screening and referral practices. Findings provide new insights about the high adaptability of programs applicable for low resource clinical settings.

\section{Background}

Food insecurity (hereafter FI) is a social determinant of health that affects approximately 33.5 million Americans(1). It is an economic condition where limited access to high-quality, nutritious food prevents individuals from leading active and healthy lives (1). Regular consumption of poor quality food contributes to poor dietary health and a high prevalence of chronic illnesses, such as obesity, diabetes, cardiovascular disease, and difficulty with disease management (1). U.S. households most affected by Fl are low-income, ethnic and minority communities, especially those affected by unemployment and job loss (2).

\section{Chronic underuse of emergency food assistance programs}

Research shows that $\mathrm{Fl}$ and poor health outcomes are related to chronic underuse of evidence-based programs, such as federal benefits programs(e.g. the Supplemental Nutrition Assistance Program (hereafter SNAP), the largest federally funded welfare program (3), and local 
emergency food pantries. Typically, stigma is associated with using welfare programs, as well as limited awareness about existing programs and complex enrollment processes $(1,4-6)$.

\section{Food insecurity screening and linkages to emergency food resources in primary care practice}

Partnerships between healthcare systems and local food assistance programs that identify and link FI patients to food assistance programs have recently emerged (7). Among those that exist, program implemented in primary care settings demonstrate the most potential to address FI because it is oftentimes revealed during routine primary care visits (8). Here, a clinician is responsible for screening patients with the application of the validated Hunger Vital Signs ${ }^{\mathrm{TM}}$ tool. Positively screened patients should be referred to emergency and long-term food assistance programs provided by local food assistance partners and wrap around services (9).

\section{Lack of theoretically derived standard practices}

FI screening and referral practices seem to be largely guided by broad national screening and referral principles that have been interpreted in many ways. This is perhaps due to their rapid and organic evolution to fill growing FI needs, yet we have limited information about program implementation and effectiveness. The emerging evidence indicates that programs and patient populations vary greatly. Therefore, it stands to reason that program activities, actors, implementation processes and outcomes also vary (9). However, our theoretical understanding about program processes is limited, and standard practices have yet to be identified. This ultimately limits efficacy testing and program replication on a broader scale. The limited evidence about implementation processes suggests that we need to study how these programs operate in the real-world setting to begin developing implementation guidelines.

\section{Implementation science}

In implementation science, theory derived frameworks have been used in healthcare research to hypothesize how system wide, clinical factors and/or multisector demands (i.e. local policies, and community needs) interact and determine the quality of implementation outcomes (10). These contextual variables can be tested in other settings where findings may lead to necessary program adaptations and wide-scale dissemination (10).

This is an opportunity to apply implementation science in the context of clinical Fl initiatives because theoretical implementation underpinnings have yet to be explored. Ultimately, findings cancan be translated into other healthcare settings $(10,11)$.

\section{Consolidated Framework for Implementation Research}

The Consolidated Framework for Implementation Research (hereafter CFIR) is an

Implementation determinants framework comprised of theoretically derived domains and constructs as seen in Table 1. CFIR has been empirically tested and is widely used in healthcare settings to understand multidimensional, interrelated implementation barriers and facilitators within specific healthcare organizations (12-14).

When used to map implementation factors, CFIR can help researchers establish a foundation from which semantic relationships between implementation factors can be constructed and tested. The framework is made up of implementation drivers that are categorized into five domains: 1) Intervention characteristics that point to the quality of the program, compatible design, its cost and adaptability across settings.

2) Inner setting, which directly relates to the physical and cultural setting where daily program processes occur. 3) Outer setting, which refers to any factor external to the program itself, including community needs, influences, local mandates, policies or regulations that affect implementation processes. 4) Characteristics of program individuals, including their knowledge and beliefs about the program. 5) Implementation processes, or the steps used in planning, execution and ongoing management of the program (10).

The purpose of this study was to understand implementation processes and outcomes of Fl screening initiatives implemented in diverse primary care practices and to identify commonalities across settings. Findings from this study were used to develop theoretically derived recommendations that may guide the development of standard practices in a systematic way.

Table 1 Consolidated Framework for Implementation Research (10) 


\begin{tabular}{|c|c|c|}
\hline CFIR Domains & Definition & Associated Constructs \\
\hline 1. Inner Setting & $\begin{array}{l}\text { Contextual environment where implementation occurs that affects } \\
\text { implementation processes }\end{array}$ & $\begin{array}{l}\text { - } \\
\text { Ctructural } \\
\text { Characteristics } \\
-\quad \text { Networks and } \\
\text { Communications } \\
-\quad \text { Culture } \\
-\quad \text { Implementation Climate } \\
-\quad \text { Readiness for } \\
\text { Implementation }\end{array}$ \\
\hline 2. Outer Settings & $\begin{array}{l}\text { Influences on implementation that are external to the intervention and/or the } \\
\text { setting within which implementation occurs }\end{array}$ & $\begin{array}{l}\text { - } \quad \text { Cosmopolitanism } \\
\text { Incentives } \\
\text { - } \quad \text { Patient Needs and } \\
\text { Resources } \\
\text { - } \quad \text { Peer Pressure }\end{array}$ \\
\hline 3. Process & Implementation activities that facilitate implementation & $\begin{array}{ll}- & \text { Engaging } \\
- & \text { Executing } \\
- & \text { Planning } \\
- & \text { Reflecting and Evaluating }\end{array}$ \\
\hline $\begin{array}{l}\text { 4. Individuals } \\
\text { Involved }\end{array}$ & Actions and behaviors of individuals actively involved in implementation & $\begin{array}{l}\text { - Individual Identification } \\
\text { with Organization } \\
\text { - Individual Stage of } \\
\text { Change } \\
\text { - Knowledge and Beliefs } \\
\text { about the Intervention } \\
-\quad \text { Other Personal Attributes } \\
-\quad \text { Self-efficacy }\end{array}$ \\
\hline $\begin{array}{l}\text { 5. Intervention } \\
\text { Characteristics }\end{array}$ & Characteristics of the intervention that result in successful implementation & $\begin{array}{ll}- & \text { Adaptability } \\
- & \text { Complexity } \\
- & \text { Cost } \\
-\quad & \text { Design Quality and } \\
\text { Packaging } & \\
-\quad \text { Evidence Strength and } \\
\text { Quality } \\
-\quad \text { Intervention Source } \\
-\quad \text { Relative Advantage } \\
-\quad \text { Trialability }\end{array}$ \\
\hline
\end{tabular}

\section{Methods}


Within the study setting in Cook County, IL, a complex healthcare community that serves 5.2 million residents with diverse health issues exists. Over 37 hospitals, several dozen Federally Qualified Health Centers (hereafter FQHCs), six certified local health departments, and nearly 100 regional and community-based organizations work to improve population health through a statewide, Alliance for Health Equity collaborative (hereafter AHE).

Two FI screening and referral programs were selected for this study using criterion sampling from a larger sample of FI screening initiatives that were identified from a previous AHE environmental scan. The scan identified 13 programs implemented within primary care settings in urban and suburban Cook County, IL. The two programs selected for this study differed in the type of setting (i.e. one public, government funded organization, the other an academic medical center). Distinct program differences allowed for the exploration of program implementation in different contexts and the extraction of common, overarching implementation themes.

Inclusion criteria were based on previous research and national recommendations for

clinical FI screening initiatives(7,15-17). Study cases met the following criteria: 1) Programs that utilized the standardized two question Hunger Vital Signs tool; 2) Programs that incorporated a referral system for food delivery; 3 ) Programs that incorporated a referral system for SNAP enrollment; 4) Programs that had been implemented for a minimum of one year. The last criterion allowed for the examination of programs that had been presumably functioning long enough that initial challenges common to start-up programs had already been addressed. The focus of this study was to explore ongoing issues that hindered or assisted implementation and sustainability.

Three programs fit the established study criteria. One program voluntarily opted out of the study. Therefore, two cases participated in this study-hereafter, Program A and Program B.

Table 2 Characteristics of study cases

\begin{tabular}{|c|c|c|c|c|c|c|}
\hline Cases & $\begin{array}{l}\text { Characteristic of } \\
\text { Healthcare } \\
\text { Organization }\end{array}$ & $\begin{array}{l}\text { Program } \\
\text { Funding }\end{array}$ & $\begin{array}{l}\text { Food } \\
\text { Organization }\end{array}$ & Location & Initiative & $\begin{array}{l}\text { Stage of } \\
\text { Implementation }\end{array}$ \\
\hline $\begin{array}{l}1 . \\
\text { Program } \\
\text { A }\end{array}$ & $\begin{array}{l}\text { Public, government } \\
\text { funded healthcare } \\
\text { system }\end{array}$ & None & $\begin{array}{l}\text { Local food } \\
\text { bank }\end{array}$ & $\begin{array}{l}\text { Urban } \\
\text { setting }\end{array}$ & $\begin{array}{l}\text { Food security screening, mobile } \\
\text { food truck, enrollment/referral to } \\
\text { benefits program }\end{array}$ & $\begin{array}{l}\text { Full } \\
\text { Implementation } \\
\text { (1 year and } \\
\text { beyond) }\end{array}$ \\
\hline $\begin{array}{l}2 . \\
\text { Program } \\
\text { B }\end{array}$ & $\begin{array}{l}\text { Private, academic } \\
\text { medical center }\end{array}$ & $\begin{array}{l}\text { Federal } \\
\text { funding and } \\
\text { local grants }\end{array}$ & $\begin{array}{l}\text { Urban } \\
\text { garden } \\
\text { collective }\end{array}$ & $\begin{array}{l}\text { Suburban } \\
\text { setting }\end{array}$ & $\begin{array}{l}\text { Food security screening, onsite } \\
\text { food distribution, enrollment } \\
\text { to benefits program }\end{array}$ & $\begin{array}{l}\text { Full } \\
\text { Implementation } \\
\text { ( } 1 \text { year and } \\
\text { beyond) }\end{array}$ \\
\hline
\end{tabular}

\section{Study design}

An embedded multiple case study design was used to examine the phenomenon of primary care situated FI screening and referral processes $(18,19)$. The embedded nature of this study refers to the multiple units of analysis within each case (19). Preliminary research for this study indicated that the healthcare context drove how FI screening programs were implemented and what types of food assistance programs were incorporated. Implementation challenges at multiple levels within the healthcare setting, such as clinicians at the micro, clinic level were examined. Program resources, technology and infrastructure, were identified as implementation barriers at the macro, systems level. Therefore, each case in this study was identified as one individual screening initiative and the units of analysis were organization wide program actors within the healthcare setting as illustrated in Figure 1.

\section{Participants}

Over the course of six months, an iterative sampling approach was used to recruit participants for this study from a convenience sample of implementation actors at each case until data saturation was achieved $(\mathrm{N}=19)$. The individual that led implementation at each case was recruited first to learn more about each program's concept, design and execution. Implementation leaders provided rich data about the implementation context representative of the following CFIR concepts: intervention characteristics, the inner setting, the outer setting, key individuals involved in the program, as well as critical implementation processes and program outcomes. Through a purposive sampling 
process, implementation leaders were asked to identify and recruit additional key actors within the healthcare organization critical to program implementation that could best inform the study with their experiences (20).

\section{Study instrument and data collection}

The interview guide used for this study was developed using the adapted CFIR framework (see Additional File 1). The guide was tailored to the program role of each implementation actor that was interviewed. As in similar research, the questions broadly asked about program activities, implementation processes, program outcomes and about major challenges/facilitators that affected feasibility and fidelity of program implementation (21).

A trained qualitative researcher (ST) conducted semi-structured, key informant interviews for this study. The interviews were face-to-face at each program site or over the telephone at the study participant's discretion. Each interview lasted 30 - 45 minutes and were audio recorded for data analysis purposes. Participants recruited for the study were made aware of the audio recording at the beginning of each session and were required to provide verbal consent prior to participation in the study. This study was approved for a claim of exemption (Protocol \# 2019-0610) from an academic Institutional Review Board on August 30, 2019.

The researcher took detailed notes during each interview that provided initial insights to the study. Revisions to the instrument guide were made after each interview for clarity and to collect additional program details.

\section{Data collection, coding and analysis}

Data were collected, managed and analyzed concurrently over a period of seven months until data saturation was achieved. Transcriptions of the interviews were uploaded to Atlas.ti v.8 Qualitative Data Analysis Software for data management, coding and assistance with analysis. All personal identifying information was removed from the data prior to analysis. All data were stored on a password protected computer only accessible by the researcher. A coding system developed a priori based on the adapted CFIR framework for data interpretation was used during data collection and analysis (see Additional File 2). Codes were added to or removed from the codebook as new ideas and concepts emerged, illustrated in Figure 2.

CFIR was adapted to fit the study context based on previous research (9). "Patient Needs," originally categorized under the construct, "Outer Setting," were found to span both the "Outer Setting" and the "Inner Setting," as it related to how the program met patient needs. Therefore, the "Patient Needs" category was placed in a separate domain as its own construct to account for the high frequency and diversity of patient issues that were reported during patient visits, as well as before and after their visits. Operational codes, such as screening, referral and program enrollment, also needed to be accounted for during the coding process and were added into the "Design" category of the "Intervention Characteristics" domain.

In addition, the "Inner Setting" domain was broken out into organizational levels during data collection. The system level, regional level, department level and/or clinical level were added to the framework to understand how macro, meso and micro levels within each organization affected implementation processes. Other healthcare studies that applied the CFIR framework for practice-based research similarly divided the inner setting into hierarchical levels to evaluate how factors at each level affected implementation of patient-centered programs (22). Based on organizational theory, this process allows researchers to understand the different levels of change that need to occur within a healthcare organization for a new innovation to be implemented effectively (23). We wanted to also understand if programs achieved fidelity through current implementation processes what barriers or facilitators emerged. Therefore, "Fidelity" was added to the "Execution" construct under the "Process" domain.

Two experienced PhD level university students (ST and LC) established interrater reliability of the coding process until 80 percent agreement was achieved as recommended for qualitative research (24).

As data were collected, memos were used to document progress, study decisions and emerging themes (25). Matrices and frameworks were developed to guide thematic analysis and anchor emerging concepts to specific CFIR constructs (26). The themes and patterns that emerged from each interview were compared to previous interview findings. This allowed the identification of commonalities, disparities and outliers in the data and for a rich understanding of program implementation to emerge (18).

For each case, program activities, time of occurrence and implementation actors were confirmed. Implementation processes were also described as originally intended, as well as unanticipated implementation facilitators and challenges and the unique implementation context that resulted in program adaptations. 
Program outcomes were also collected to assess implementation feasibility, effectiveness, as well as overall program fidelity (21,27). The following program outcomes were identified across cases: the number of patients screened; the number that identified as Fl; the number of patients referred to food assistance programs; the number of patients that participated in the food assistance program. The frequency that clinicians completed essential program activities was also collected to tie outcomes specific program elements. During the cross-case analysis, the binding implementation themes were identified and gave meaning to program outcomes.

Atlas.ti v8 exploratory functions were used to further analyze and confirm findings, as was source triangulation between participants. The data were mined specifically for statements that juxtaposed with each program activity, outcome and implementation processes. This method allowed for a clearer picture about specific facilitators/challenges and brought clarity to implementation processes. Overlap between constructs helped to establish the semantic relationships between CFIR constructs. Prior to the finalization of study results, one program leader and one clinician from each case were asked to participate in member-checks. They each reviewed the results from their respective case, and provided feedback where necessary to ensure validity of study findings.

\section{Results}

Study findings revealed intended program activities, actors, processes and outcomes specific to each case, illustrated in Table 3 and Table 4.

Table 3 Description of intended activities and actors of each program 


\begin{tabular}{|c|c|c|}
\hline Case & Intended Program Activity & $\begin{array}{l}\text { Intended } \\
\text { Implementation } \\
\text { Actor }\end{array}$ \\
\hline \multirow[t]{2}{*}{$\begin{array}{l}\text { Program } \\
\text { A }\end{array}$} & 1. EHR FI screening once a year during patient intake & $\begin{array}{l}\text { Medical } \\
\text { Assistant }\end{array}$ \\
\hline & 2. Refer FI patients a fresh produce truck during doctor's & Physician \\
\hline
\end{tabular}

visit with a voucher to receive free produce

3. Referral to local food assistance resources during doctor's visit Physician

4. SNAP enrollment right after doctor's visit Social Worker

5. Phone call reminder to voucher recipients one to two weeks prior to the day of food distribution Social Worker

6. Food distribution once every two months through a fresh produce truck parked outside clinic Social Worker in collaboration with food partner

7. Evaluation by collecting food truck participation rates through voucher redemption Social Worker

Program 1. Screening during collection of patient vitals during routine doctor's visit; positive screen flagged in Nurse or B the EHR Medical Assistant

2. Referral to local food assistance resources during doctor's visit

Physician

3. Referral to produce prescription program

Physician

4. Call FI to enroll in the produce prescription program

Program

Manager

5. The Produce Prescription Program was held weekly

Program

during a two-hour window, one evening per week that included nutrition education classes

Manager

6. SNAP eligible patients could enroll in SNAP using an

Program

electronic tablet provided by the clinic

Manager

7. A weekly patient satisfaction survey distributed to patients after program participation. Questions asked about food preferences, cooking and nutrition lessons. Every fifth session clinical staff distributed a survey to measure change in FI status or improvements in dietary behavior due to program participation.

Table 4 Program outcomes 


\begin{tabular}{|c|c|c|c|c|}
\hline \multirow[t]{2}{*}{ Program Activity } & \multicolumn{2}{|l|}{ Program A } & \multicolumn{2}{|l|}{ Program B } \\
\hline & $\begin{array}{l}\text { Number of } \\
\text { Patients* }\end{array}$ & $\begin{array}{l}\text { Proportion of patient } \\
\text { population treated per } \\
\text { month }\end{array}$ & 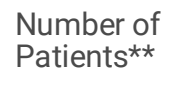 & $\begin{array}{l}\text { Proportion of patient } \\
\text { population treated per } \\
\text { month }\end{array}$ \\
\hline $\begin{array}{l}\text { Intended number of patients screened for } \mathrm{FI} \\
\text { (patient population served per month) }\end{array}$ & 1,250 & $100 \%$ & 453 & $100 \%$ \\
\hline Actual number of patients screened for $\mathrm{Fl}$ & 1,250 & $100 \%$ & 326 & $72 \%$ \\
\hline Number of patients screened positive for $\mathrm{FI}$ & 300 & $24 \%$ & 140 & $31 \%$ \\
\hline $\begin{array}{l}\text { Number of patients referred to the food } \\
\text { assistance intervention }\end{array}$ & 300 & $24 \%$ & 140 & $31 \%$ \\
\hline Number of patients received resource list & $\begin{array}{l}\text { Data } \\
\text { unavailable }\end{array}$ & Data unavailable & $\begin{array}{l}\text { Data } \\
\text { unavailable }\end{array}$ & Data Unavailable \\
\hline Number of Social Worker visits to manage FI & $\begin{array}{l}\text { Data } \\
\text { unavailable }\end{array}$ & Data unavailable & N/A & N/A \\
\hline $\begin{array}{l}\text { Number of times clinicians made phone call } \\
\text { reminders to patients }\end{array}$ & 100 & $8 \%$ & N/A & N/A \\
\hline $\begin{array}{l}\text { Number of patients that participated in the } \\
\text { food assistance intervention }\end{array}$ & 167 & $13 \%$ & 115 & $25 \%$ \\
\hline Number of patients enrolled in SNAP benefits & 0 & 0 & 0 & 0 \\
\hline \multicolumn{5}{|c|}{$\begin{array}{l}\text { *Because Program A included } 3 \text { study clinics, outcomes were averaged across the three study clinics at one point in time. Program A } \\
\text { tracked program activities from the start to the end of each month. }\end{array}$} \\
\hline
\end{tabular}

In Program A, all patients $(n=1,250)$ at each study clinic $(n=3)$ were screened yearly for FI using the EHR system during intake and followed up again the subsequent year. Based on previous community-wide data, $45 \%$ of clinic patients $(n=563)$ were $\mathrm{Fl}$ and should have been identified through screening (28). $24 \%$ of patients $(n=300)$ screened positive and were given a voucher that could be redeemed for free food at a fresh produce truck parked outside the clinic once every two months. Phone call reminders that should have been made to all FI patients were made to an average of $n=100$ patients at each clinic as a reminder about participation in the food truck program, and $n=167$ patients on average participated in the food truck program per clinic. During the doctor's visit, Fl patients were to receive a local food assistance resource list and then see the Social Worker to enroll in SNAP if eligible. These data were tracked in the EHR system and were unavailable for this study due to patient privacy concerns.

In the Program B study clinic $(n=1)$, the average number of patients served per month was $(n=453)$. Also based on community-wide data, a potential of $204(45 \%)$ of patients could have been identified as FI during screening (28). All patients were supposed to be screened for $\mathrm{FI}$ using a self-administered paper survey distributed at patient check-in and collected by the nurse that roomed the patient. 326 patients (72\%) were actually screened. Of those patients, $n=140(31 \%)$ of the clinic patient population screened positive for $\mathrm{Fl}$ and were referred to a 6 month long, produce prescription program that distributed a box of free seasonal produce every week and that also provided cooking demonstrations and nutrition education. $25 \%$ of patients $(n=115)$ participated in the food distribution program. During the doctor's visit, $\mathrm{FI}$ patients were to receive a local food assistance resource list from their doctor. These data were tracked in the EHR system and were unavailable for this study due to patient privacy concerns. Neither case enrolled patients in SNAP benefits as intended.

\section{Limited healthcare resources as a barrier}

As a part of the "Inner Setting" domain, physical space was identified as a resource challenge for both programs at the clinic level. Rigid workflow processes a part of the "Inner Setting" domain at the systems level resulted in a screening process that could only take place during the initial patient intake. For Program A, intake lacked privacy due to limited physical space. Multiple clinicians conducted intake simultaneously only a few feet apart at the "nurse's station." Clinicians revealed that patients experienced discomfort when presented with FI questions and commonly denied experiencing $\mathrm{Fl}$, even if that was not the case. This could explain why a lower than expected number of $\mathrm{FI}$ patients were identified across cases. 
For Program B, limited space and equipment at the clinic for evidence-based cooking and nutrition classes allowed for a maximum of 30 patients to participate in the program-less than half of identified FI patients

Implementation actors' capacity was also revealed as a resource challenge. Each program had challenges related to staff time, a product of existing workflow processes in both cases. Limited staff time, as a part of the "Characteristics of Individuals" domain, was a system-wide problem that fit within the larger "Inner Setting" domain. Clinicians consistently reported that limited time with patients and other high priority health issues prevented them from providing the additional food assistance resource list. Program A social workers also reported that phone call reminders were time consuming and could be performed about a third of the time. This finding maybe correlated with the low number of patient participation in the food truck program.

The Electronic Medical Records (EMR) software was also problematic across cases stemming from the system-wide "Inner Setting" domain. For Program A, screening frequency was limited to the pre-programmed system, where FI screening was prompted once a year and during intake. Clinicians reported that this was not frequent enough to track the episodic and cyclical nature of $\mathrm{Fl}$, and could not be changed.

Clinicians from Program B reported that they had difficulty navigating their EMR system. The food resource list was embedded in the system that clinicians could not find it within the system. Program B was also forced to use a paper FI screening form rather than the originally intended EHR system because it was inaccessible for this purpose due to system upgrades at the time of this study. The inherent weakness of self-administered, paper tools for the collection, analysis and storage of patient data may explain why not every patient at Program B's clinic were screened.

Limited financial resources, associated with the "Inner Setting" domain negatively affected both programs as it related to frequency of food distribution, program sustainability and reach. Neither program had the financial means to pay for a realistic, long-term solution to FI. Program A relied on in-kind donations from their food assistance partner that could only be provided once every two months. Program $B$ relied on their food partner's established infrastructure for food distribution-a local gardening collective during the 6-month growing season. Moreover, because Program B's funding was supplied by the USDA, federal spending restrictions prevented SNAP-ineligible patients from program participation.

\section{Leveraging the unique implementation context as a facilitator}

Both programs used their existing external partnerships and/or the internal work culture to facilitate implementation. The community climate -cosmopolitanism as a part of the "Outer Setting" domain-played a big role in Program B planning and execution. The community environment fostered food justice for almost a decade prior to Program B's conception. Multi-sector networks supported an urban growing model that promoted local, seasonal and affordable produce for distribution in low-income households. Program B's inherent synergy with existing community initiatives gave it access to an existing program model, expertise and infrastructure. The program did not have to be built from scratch. The local food justice organization, which advocated for economic development, health equity and sustainable food system played a fundamental role in bringing together the health system, local food growers and other health and wellness community organizations. Their collective strength supplemented the initial program design to improve reach and impact. One major adaptation was to utilize the existing partnership with the parks department to shift program activities from the clinic to the local community center. The space could accommodate more people, tripled patient participation and fostered social support and community engagement. During year two of the program, intervention leaders secured additional grant funding through another local partner that allowed for the purchase of additional food from local growers for year-round food distribution and to fill the gaps in services related to SNAP eligibility. As a result, patient participation in the program more than tripled.

The community context also fostered an aligned internal implementation climate for the uptake of Program B by clinicians. The implementation climate, associated with the "Inner Setting," systems level domain and the climate construct, included several existing farm stands across the health system campus. Clinicians said it was common for patients to come to their appointments with their shopping bags of produce from the stand, which prompted a dialogue about diet and nutrition for disease management.

The aligned climate was also observed in the academic side of Program B's healthcare organization. Medical and dietetics students were tapped as additional program staff support. The cross-departmental collaboration trained students to implement the cooking demonstrations and nutrition education components, which helped to mitigate the lack of program staff that was a problem from the start.

Program A leveraged its culture of clinic-level autonomy to facilitate implementation. Clinic managers were given decision-making authority to implement activities as they saw fit. The program leader indicated that, "Managers need to take ownership because they know their patients and their staff." In turn, clinic managers reported specific adaptations as demonstrated through statements of, "We do things differently here," and "the way we do it is..." Statements of this kind referred to linkages and voucher activities that clinicians said they

Page 10/19 
adapted to better suit the needs of their patients. Clinicians said that vouchers were distributed universally, to reduce the stigma of singling out patients during FI screening and helped foster trust with their patients. This adaptation could explain why the number of patients that participated in the food truck program was 1.5 times greater than the number of reminder phone calls made. Another clinic adapted the SNAP enrollment process because there was not enough time for enrollment during patient visits. Instead, clinicians said they engaged patients while they waited in the food truck line, which facilitated enrollment. These data were not available for this study. Adaptability and trialability of program characteristics

Both programs were highly adaptable and testable-two constructs associated with the "Intervention Characteristics" domain. Adaptability refers to the degree to which the core program components can be tailored to fit the implementation context (10). Trialability refers to the ability for stakeholders to pilot an intervention on a small scale and engaged in quality improvement efforts (10). Both constructs emerged in the context of limited program resources. It appeared that when limited program resources were supplemented, high levels of adaptability and trialability characteristics were revealed. Conversely, the high level of adaptability and trialability of each program allowed for ongoing exploration of alternative and creative methods to improve program implementation, reach and sustainability.

Table 5 lists the overarching study themes and illustrative quotes that assisted in the interpretation of findings.

Table 5 Overarching study themes and illustrative quotes 


\begin{tabular}{|ll}
\hline Theme & Illustrative Quotation \\
\hline $\begin{array}{l}\text { Limited healthcare } \\
\text { resources/EMR }\end{array}$ & $\begin{array}{l}\text { "IT has not been very helpful because they have not been involved with our program so we're still using } \\
\text { paper surveys... we only have one person in charge of screening and she's overwhelmed..." - Clinician, } \\
\text { Program B }\end{array}$ \\
$\begin{array}{l}\text { Limited healthcare } \\
\text { resources/EMR }\end{array}$ &
\end{tabular}

resources/EMR

Limited healthcare

resources/space

Leveraging context (cliniclevel autonomy) to foster implementation adaptations

"It's up to the clinic staff to follow through with how they do this because we respect the autonomy of each clinic to take responsibility for the program and how it's done." -Program Leader, Program A

"Screening for food insecurity once a year isn't enough, but that's how our EHR works." Clinician, Program

"The food insecurity questions are not embedded in the EHR...the paper system is inefficient for assessing food insecurity needs across the board."

-Clinician, Program B

"Unfortunately, it's (screening) not very private. And what I mean by that is that it's open to more medical assistants that are sitting at that station and potentially another patient getting vitals next to them...So as they're asking them the questions, there are more people around and it's not very private. Sometimes we do have that response of no, no, no I'm fine and then they get inside and they tell the doctor maybe something different."

-Clinician, Program A

"Yeah, so anyone can get a voucher that's a patient. How we do it is that even after the nurses give them out, we give vouchers to the clerks and other staff that may run into patients. A patient may come up just to pick up some documentation and be like, 'Oh, I know the Fresh Food Truck has come in. Can I have a voucher?' Everyone has access to the vouchers so they can give them out at any time on any day." Clinician, Program A

"In general, food insecurity has not been on my radar... As you probably know, a primary care visit is completely overwhelming with-'I have to get your foot exam done, you have to get an influenza shot, I've got to draw your A1C.' Food insecurity is a serious conversation that takes time...And so that is why it has not been a part of my standard practice." - Clinician, Program A

“We don't have a way of flagging patients that are food insecure in the EHR to remind doctor's to talk to patients about resources and sometimes they forget" - Program Leader, Program A

Limited healthcare resources/staff capacity

"So we used to have a [phone call] list of the patients who answered yes to the food insecurity questions, but [the phone call reminders] didn't work with us, and was very ineffective because a lot of times patients say no [when asked if they experience food insecurity], but it's really yes. So their name wouldn't be on the list. So we just wiped out the list altogether and emphasize 'don't lose your voucher."' - Clinician, Program A 


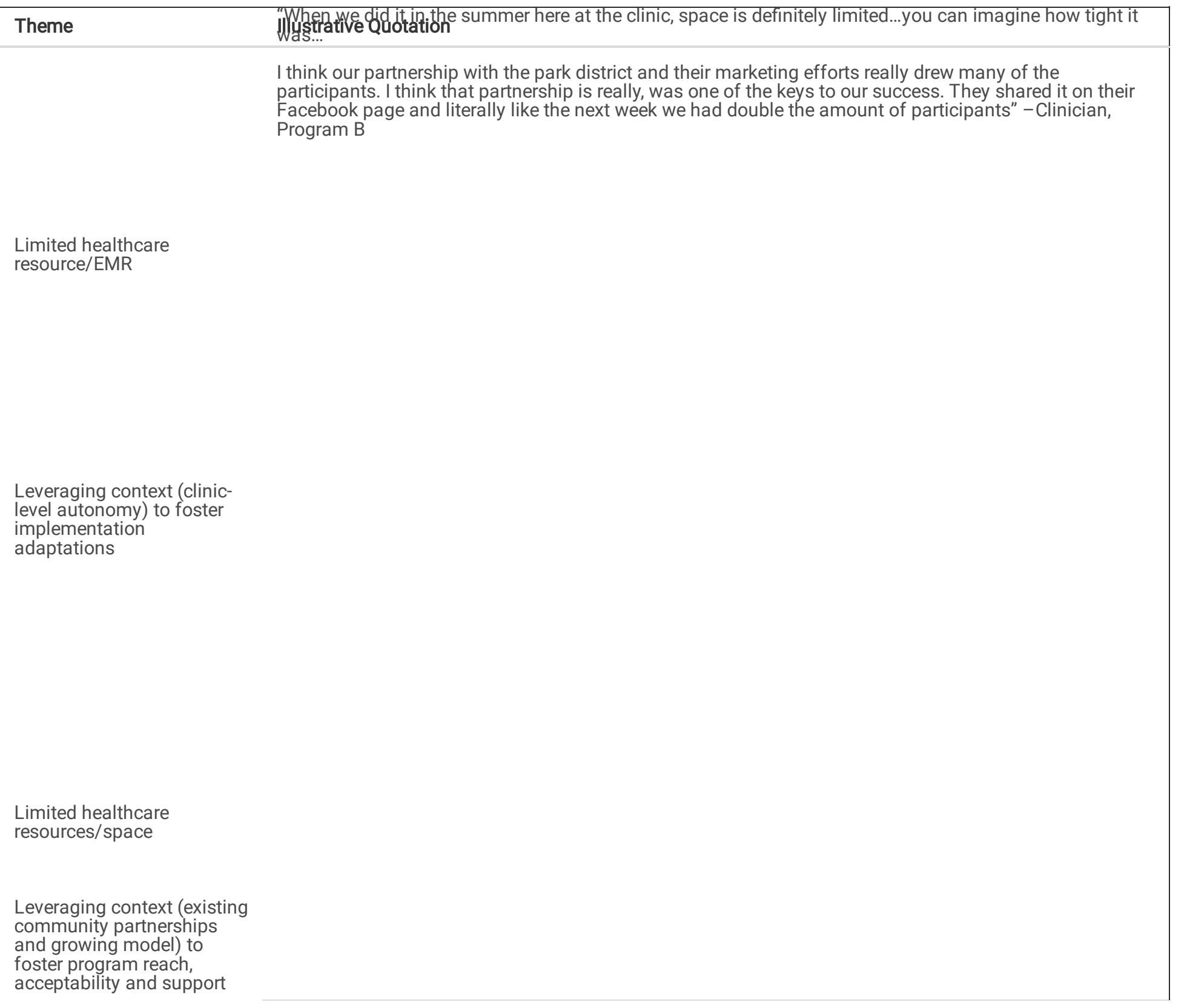

Limited healthcare resources/financial

"The food truck visits at each site are not frequent enough because it requires an incredible amount of [food pantry] capacity to staff to run the truck and we don't have the funds to pay for that. Bad weather, like last year's polar vortex, can deter participants from coming to the distribution...Patients want more frequent distribution." -Program Leader, Program A

"On that [USDA] grant it's a total of four [years]...I think we've also realized that it's not a sustainable solution to food insecurity. We're really trying to think of what else can we do to make this a sustainable food economy here in [neighborhood name]." - Program Leader, Program B

Limited healthcare resources/financial 


\begin{tabular}{|l|l|}
\hline Theme & $\begin{array}{l}\text { Illustrative Quotation } \\
\text { "We've been close partners with [the urban garden collective], and we wrote the USDA grant together for } \\
\text { [Program B]. So, the growing concept came from them... One, the hospital alone can't do it, but when we all } \\
\text { came together it was feasible." - Program Leader, Program B }\end{array}$ \\
$\begin{array}{l}\text { Leveraging context (existing } \\
\text { and growing model) to } \\
\text { foster program } \\
\text { acceptability and support }\end{array}$ \\
$\begin{array}{l}\text { "I think the strength of having it at the clinic is just the traffic. The traffic of people. So, people walking } \\
\text { past the farm stand and seeing the vegetable distribution get naturally brought into the cooking demos... } \\
\text { the farmstand is a brilliant move because that farmstand is sitting there just seamlessly with the }\end{array}$ \\
[prescription produce box] distribution... It becomes a very collective experience. " - Clinician, Program B \\
$\begin{array}{l}\text { Leveraging implementation } \\
\text { limate (existing growing } \\
\text { model) to foster program }\end{array}$
\end{tabular}

\section{Discussion}

Study findings indicate that the unique culture and context of each cases' healthcare system drove implementation processes and program outcomes. Each program demonstrated a high-level of adaptability and trialability that accommodated the situational context and made program implementation feasible, despite lower than expected reach and fidelity across cases.

This is the first study, to this author's knowledge, that applied CFIR to examine system-wide implementation factors of clinical FI screening initiatives within the context of healthcare settings. Empirically tested and theoretically derived concepts guided the development of a conceptual implementation model, while integrating program outcomes strengthened the interpretation of qualitative findings. The conceptual model in Figure 3 may be tested and refined in follow-up studies. The model suggests a bi-directional relationship between adaptability/trialability and enhanced resources through unconventional sources within the existing climate. These factors should be explored in other settings as a way to not only facilitate implementation, but program reach, impact and sustainability.

\section{Recommendations}

Study findings resulted in the following recommendations for health systems: 1) Allow for adaptations with caution. Unique implementation contexts can foster implementation feasibility. Yet, considerations need to be made about how adaptations may negatively impact fidelity, reach and effectiveness. 2) Consider how the context can support intervention activities through clinician input about workflow, program responsibilities and time management. 3) Conduct asset mapping and outreach to potential community partners that have a strong presence in the community, aligned goals and objectives and resources that can be leveraged during program design and implementation. This recommendation raises its own challenges about whose responsibility within the health system it is to make community-wide connections and manage relationships. 4) Consider non-traditional forms of staff support. In this study, allied health and medical students were motivated to work as interns in exchange for hands-on, experiential learning. Generally, students are subject to high turnover and may not always be the best solution to fill staffing shortages that require a long-term commitment. An alternative solution is to leverage the role and expertise of community health workers that are trusted sources of information for patients because often they are members of the communities they serve.

\section{Limitations}

1) As a study instrument, the researcher was positioned alongside study participants during the process of information discovery during data collection and analysis. As such, this was a subjective process that may have been affected by the researcher's own biases and experiences (29). The researcher utilized source triangulation and member checks to negate the effect of these factors during data analysis and interpretation.

2) The presence of the researcher during data collection may also have affected participants' responses, which could potentially undermine reliability. However, the strength of qualitative studies such as this one is that they explored practice activities within the implementation context and build on previous practice knowledge (30). Data from this study assisted with the translation of clinical practice into theoretical assertions that can be generalized in future practice-based research (30).

3) There may have been a potential disadvantage of using the cases from the AHE environmental scan for this study. Because participation in the study was voluntary and targeted at AHE participants, the sample may not have been entirely representative of all primary care 
situated programs in Cook County, especially those not connected with AHE. AHE participants were highly engaged in advocacy and policy development to address FI in local populations. The study cases came from a pool of potentially biased organizations that were highly motivated to address patient Fl. Future studies may build on these findings by exploring implementation processes within clinical settings where stakeholder buy-in is relatively low.

While this study incorporated the perspective of multiple implementation actors representative of the implementation context, the study did not include patients' perspectives.

Patient level data could have enriched the findings and strengthened the validity of findings while also contributing a unique patient experience. The amount of time allotted for this study limited the scope to implementation actors only. Future studies should incorporate patient perspectives about program implementation through a community-engaged approach.

The lack of in vivo observations and EHR data in this study could have offered a real-time representation of implementation processes that would have enhanced study findings through triangulation and, perhaps, additional insight. Time restrictions prevented the addition of observations to this study and EHR data was not accessible. Future research should incorporate direct observations and EHR data to build on current findings.

Due to time restrictions, data that were collected at only one point in time and relied on the memory of each participant. Future studies should consider the collection of data from participants at multiple time points to capture the dynamic process of implementation and to further validate findings.

\section{Implications}

This study makes significant contributions to the limited body of literature in the emerging field of clinical FI screening programs in primary care practice. In particular, the proposed conceptual model is a foundation for the development of theory-driven standard practices. Though formative in nature the model identifies areas of exploration that have not been considered in previous research, such as the internal work culture and the community climate.

Study findings have implications for practice-based research. The exploration of external factors and creative uses of internal assets for program support should be considered due to the scarcity of funding for community-based interventions implemented in low-resource clinics. Future work should consider how these factors may enhance limited internal resources long-term. Community-engaged research with patients could help to tailor primary care focused initiatives to the realities of patient needs. Engaging the patient community could provide critical insights about stigma, privacy, trust and workflow processes from the patient's perspective, as well as provide deeper understanding about the cyclical nature of household FI that may inform frequency of screening and can be used to advocate for additional services that fully meet patient needs. Study findings also have implications for ongoing policy work of universal screening practices supported by national healthcare experts

\section{Conclusion}

The key take away from this study is that due to limited resources, primary care practices that serve low-income communities need to be supported in their ability to adapt program activities to their specific context. While high program fidelity and intended program outcomes may not have been achieved in this study, findings demonstrate how implementation feasibility can be achieved when community partnerships and an internal work culture are leveraged for program support. With this in mind, future research may continue to build on the proposed conceptual model, which is formative in nature and sets the stage for development of standard screening practices. As our healthcare system continues its transition to a value-based model of care, we need to consider how primary care focused FI screening initiatives can effectively connect patients to food resources. If we can reduce the inequitable access to affordable and healthy food, we may eventually see long-term improvements in the quality of life of our most vulnerable populations.

\section{Abbreviations}

\section{$\mathrm{FI}$}

Food Insecurity/Food Insecure; SNAP:Supplemental Nutrition Assistance Program; WIC:Special Supplemental Nutrition Assistance Program for Women's Infants and Children; EHR:Electronic Health Records; EMR:Electronic Medical Records; CFIR:Consolidated Framework for Implementation Research; AHE:Alliance for Health Equity

\section{Declarations}




\section{Ethical approval and consent to participate}

All procedures in this study involving human subjects were in accordance with ethical standards of an institutional research committee and with the 1964 Helsinki Declaration and its later amendments or comparable ethical standards. Informed consent was obtained from all participants included in the study.

\section{Consent for Publication}

All participants provided verbal consent before participation in the study, including consent to publish anonymous quotations.

\section{Author's Contributions}

ST led the conception and design of the study, development of study instruments, conducted interviews, data analysis, data interpretation and led manuscript writing. NM assisted with critical revisions of the manuscript for content. AOY assisted with developing the study design, data interpretation and critical revisions of the manuscript. MF, NP, and CS assisted with data interpretation and critical review of the manuscript.

\section{Funding}

This study was funded by an academic research award funded by the researcher's academic institution.

\section{Availability of data}

Datasets from this study are not publicly available due to institutional review board regulations, but selective, di-identified and aggregated data may be made available upon reasonable request.

\section{Competing interests}

The authors declare that they have no competing interests.

\section{Acknowledgements}

The authors would like to thank all study participants for taking time out of their busy schedules to contribute to this study. We would like to thank Kathy Chan and Lena Hatchett for their assistance with recruitment.

\section{References}

1. Services USD, of AER. Food, Assistance N. Food Security in the U.S., Measurement [Internet]. Vol. 2017. 2016. Available from: https://www.ers.usda.gov/topics/food-nutrition-assistance/food-security-in-the-us/measurement/.

2. Gundersen C, Engelhard EE, Crumbaugh AS, Seligman HK. Brief assessment of food insecurity accurately identifies highrisk US adults. Public Health Nutr. 2017;20(08):1367-71.

3. Nestle M. The Supplemental Nutrition Assistance Program (SNAP): History, politics, and public health implications. Am J Public Health [Internet]. 2019 Dec 6 [cited 2020 May 28];109(12):1631-5. Available from: https://ajph.aphapublications.org/doi/10.2105/AJPH.2019.305361.

4. Browne NT. Food Insecurity: Assessment and Intervention. J Pediatr Surg Nurs [Internet]. 2017;6(1). Available from: http://journals.Iww.com/journalofpediatricsurgicalnursing/Fulltext/2017/01000/Food_Insecurity_Assessment_and_Intervention.5.aspx.

5. Gilbert D, Nanda J, Paige D. Securing the safety net: concurrent participation in income eligible assistance programs. Matern Child Health J. 2014 Apr;18(3):604-12.

6. Neff RA, Palmer AM, McKenzie SE, Lawrence RS. Fc. J Hunger Environ Nutr. 2009 Jul;4(3-4):282-314.

7. Barnidge E, Stenmark S, Seligman H. Clinic-to-Community Models to Address Food Insecurity. JAMA Pediatr. 2017 Jun;1(6):507-8. 171(.

8. Gupta DM, Boland RJ Jr, Aron DC. The physician's experience of changing clinical practice: a struggle to unlearn. Implement Sci. 2017 Feb 28;12(1):22-8.

9. Lundeen EA, Siegel KR, Calhoun H, Kim SA, Garcia SP, Hoeting NM, et al. Clinical-community partnerships to identify patients with food insecurity and address food needs. Prev Chronic Dis. 2017;14:E113.

10. Damschroder LJ, Aron DC, Keith RE, Kirsh SR, Alexander JA, Lowery JC. Fostering implementation of health services research findings into practice: a consolidated framework for advancing implementation science. Implement Sci. 2009 Aug 7;4:50. 
11. Glasgow RE, Vinson C, Chambers D, Khoury MJ, Kaplan RM, Hunter C. National Institutes of Health Approaches to Dissemination and Implementation Science: Current and Future Directions. Am J Public Heal [Internet]. 2012;102(7):1274-81. Available from: https://doi.org/10.2105/AJPH.2012.300755.

12. Damschroder LJ. Clarity out of chaos: Use of theory in implementation research. Psychiatry Res. 2020 Jan 1;283:112461.

13. Kirk MA, Kelley C, Yankey N, Birken SA, Abadie B, Damschroder L. A systematic review of the use of the Consolidated Framework for Implementation Research. Vol. 11: Implementation Science. BioMed Central Ltd.; 2016.

14. Li SA, Jeffs L, Barwick M, Stevens B. Organizational contextual features that influence the implementation of evidence-based practices across healthcare settings: A systematic integrative review. Syst Rev. 2018 May 5;7(1):1-19.

15. Garg A, Toy S, Tripodis Y, Silverstein M, Freeman E. Addressing Social Determinants of Health at Well Child Care Visits: A Cluster RCT. Pediatrics [Internet]. 2015;135(2):e296-304 1p. Available from: http://search.ebscohost.com/login.aspx? direct=true $\& \mathrm{db}=r \mathrm{zh} \& \mathrm{AN}=103794683 \&$ site=ehost-live.

16. Barnidge E, LaBarge G, Krupsky K, Arthur J. Screening for Food Insecurity in Pediatric Clinical Settings: Opportunities and Barriers. J Community Health. 2017 Feb;42(1):51-7.

17. Network N, OPR and E. Food Insecurity Screening Algorithm for Pediatric Patients [Internet]. Vol. 2017. 2017. Available from: http://frac.org/wp-content/uploads/food-insecurity-screening-algorithm-pediatric-patients.pdf.

18. Stake RE. The art of case study research. Thousand Oaks: Sage; 1995.

19. Yin KR. Case Study Research and Applications: Design and Methods. 6th ed. Thousand Oaks: SAGE Publications Inc.; 2018.

20. Creswell JW, Poth CN. Qualitative Inquiry and Research Design: Choosing Among Five Approaches. 4th ed. 100. Thousand Oaks: SAGE Publications Inc.; 2018. 148 p.

21. Gold R, Bunce A, Cottrell E, Marino M, Middendorf M, Cowburn S, et al. Study protocol: A pragmatic, stepped-wedge trial of tailored support for implementing social determinants of health documentation/action in community health centers, with realist evaluation. Implement Sci [Internet]. 2019 Jan 28 [cited 2021 Feb 26];14(1):1-17. Available from: https://doi.org/10.1186/s13012-019-0855-9.

22. Safaeinili N, Brown-Johnson C, Shaw JG, Mahoney M, Winget M. CFIR simplified: Pragmatic application of and adaptations to the Consolidated Framework for Implementation Research (CFIR) for evaluation of a patient-centered care transformation within a learning health system. Learn Heal Syst [Internet]. n/a(n/a):e10201. Available from: https://onlinelibrary.wiley.com/doi/abs/10.1002/Irh2.10201.

23. Ferlie EB, Shortell SM. Improving the Quality of Health Care in the United Kingdom and the United States: A Framework for Change. Milbank Q. 2001;79(2):281-315.

24. Pluye P, Hong QN. Combining the power of stories and the power of numbers: mixed methods research and mixed studies reviews. Annu Rev Public Health. 2014;35:29-45.

25. Patton MQ. Qualitative Research and Evaluation Methods. 4th ed. Thousand Oaks: SAGE Publications, Inc.; 2015.

26. Gale NK, Heath G, Cameron E, Rashid S, Redwood S. Using the framework method for the analysis of qualitative data in multidisciplinary health research. BMC Med Res Methodol [Internet]. 2013;13:117. Available from: https://www.ncbi.nlm.nih.gov/pubmed/24047204.

27. Haynes A, Brennan S, Redman S, Williamson A, Gallego G, Butow P. Figuring out fidelity: A worked example of the methods used to identify, critique and revise the essential elements of a contextualised intervention in health policy agencies. Implement Sci [Internet]. 2016 Feb 24 [cited 2021 Apr 1];11(1):23. Available from: http://www.implementationscience.com/content/11/1/23.

28. County HIC of C. Community Needs Assessment Central Region CHNA. Chicago, IL: Illinois Institute of Public Health; 2016.

29. Stake RE. Multiple Case Study Analysis. New York: The Guilford Press; 2006.

30. Miles R. Complexity, representation and practice: Case study as method and methodology. Issues Educ Res [Internet]. 2015;25(3):30918. Available from: http://www.iier.org.au/iier25/miles.html.

\section{Figures}




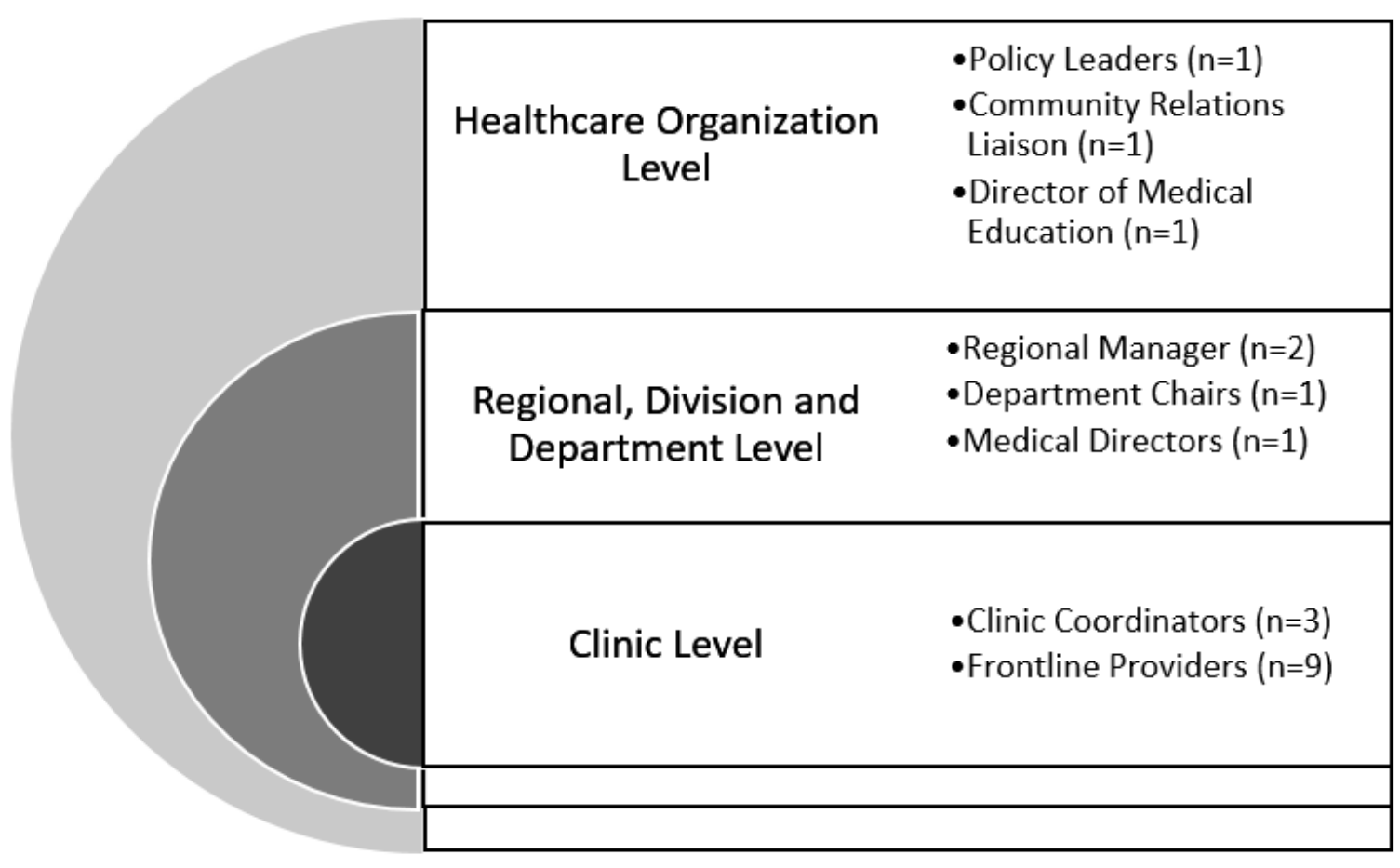

Figure 1

Units of analysis across healthcare organizations in this study ( $\mathrm{N}=19)$

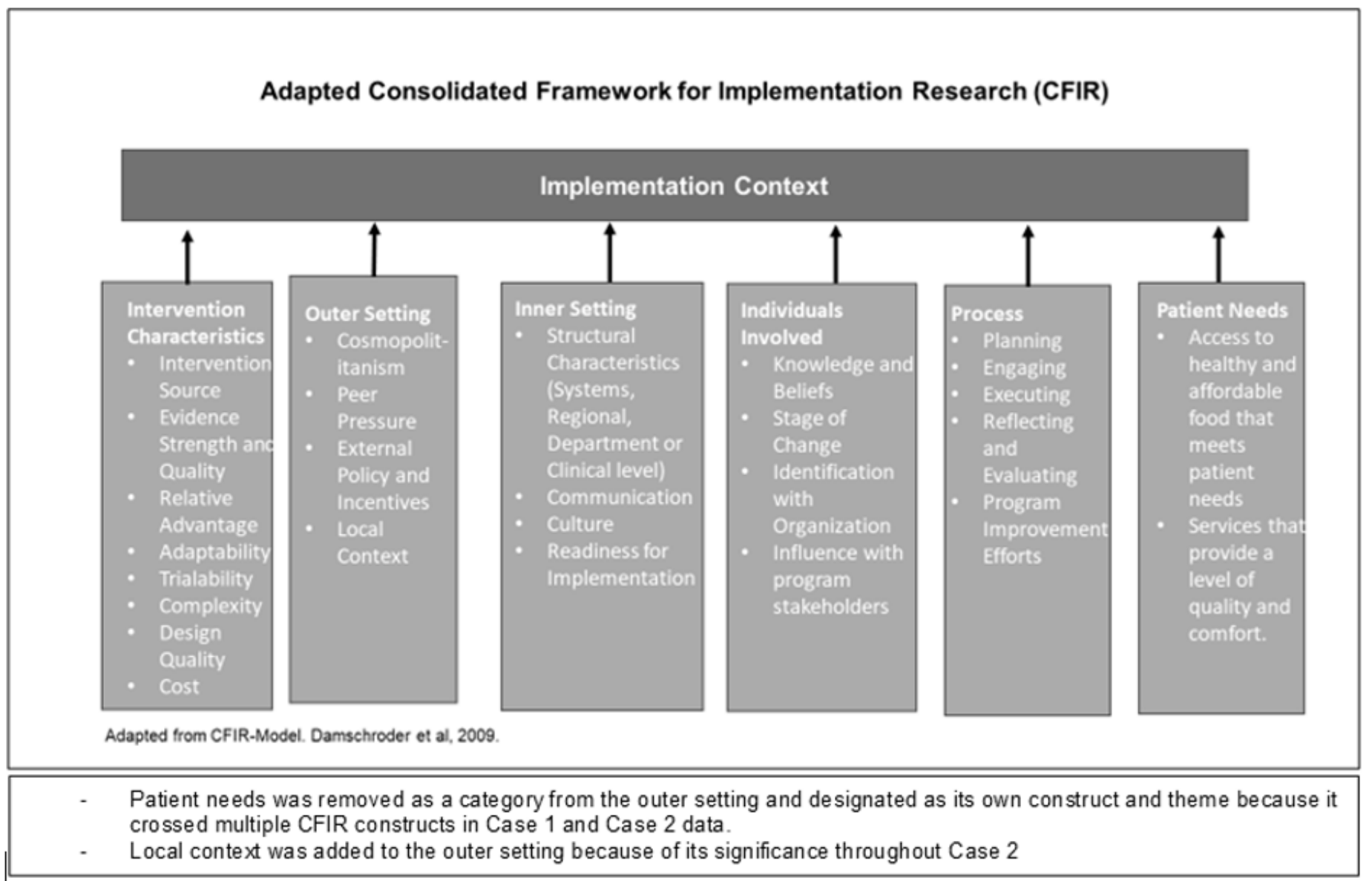

Figure 2

Adapted CFIR framework for this study 


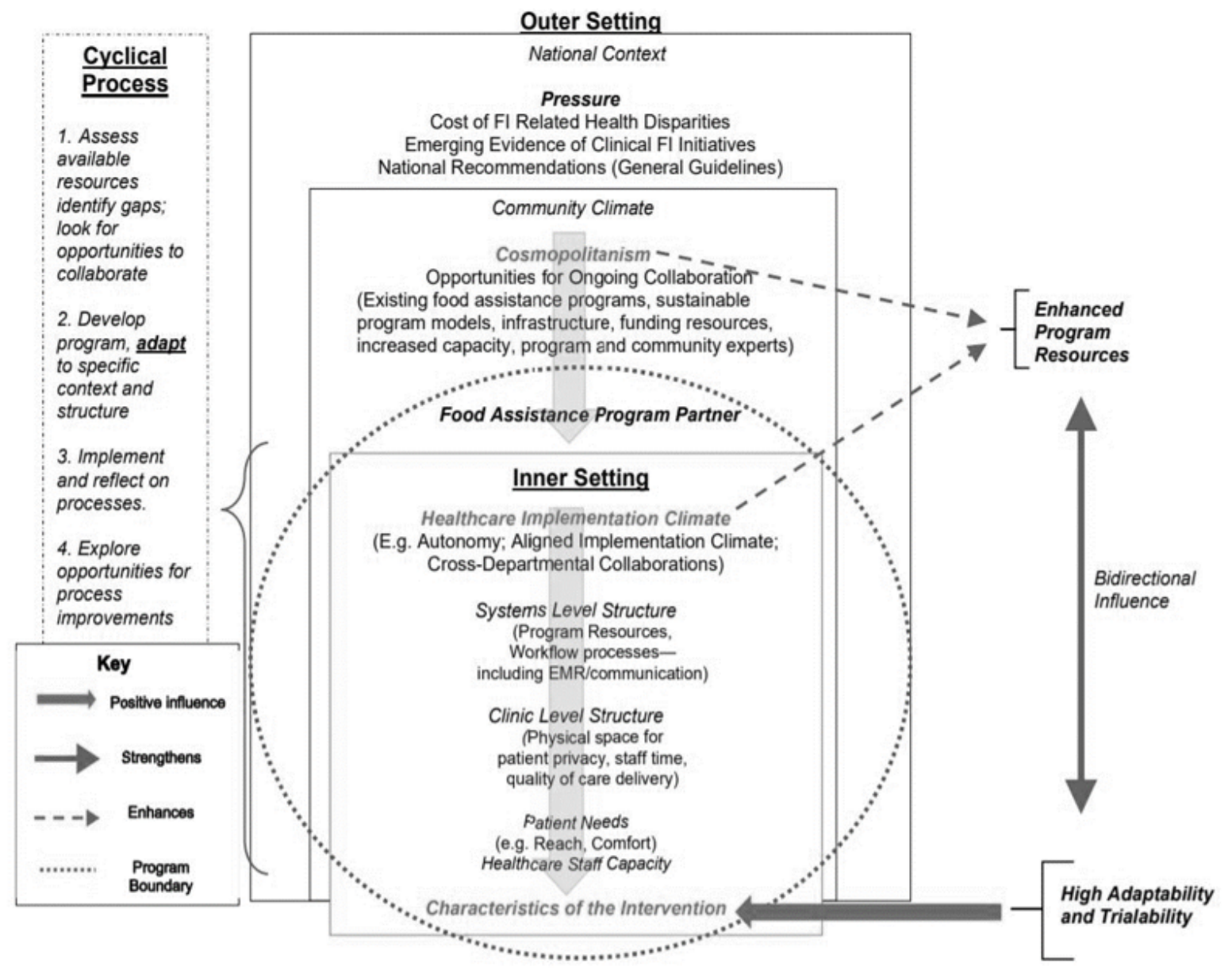

Figure 3

Conceptual model for implementation of food insecurity screening programs in primary care practice

\section{Supplementary Files}

This is a list of supplementary files associated with this preprint. Click to download.

- 04.26.21SRQRImpISci.docx

- AdditionalFile1.docx 